\title{
Radio-manométrie trans-hépato-vésiculaire
}

La radio-manométrie biliaire issue de la cholangiographie de Mirízzi, telle qu'elle est mise au point, avec des techniques diffé-rentes, dans les services de Mallet-Guy de Lyon, et Carolí de Paris, a rendu le plus grand service aux patients, en fournissant aux chirur-giens une appreciation plus precise de la situation et, par consequent, en dirigeant mieux leur decision opératoire. Bien qu'elle soit pratiquée maintenant au cours même de l'intervention, au lieu d'exiger l'établissement préalable d'une eholécystostomie et un second temps opératoire éventuel, la radiomanométrie n'était pas possible, jusqu'ici, sans laparotomie. Certes, Royer, de Buenos-Aires, ponctionne la vésicule sous contrôle laparoscopique, mais celui-ci exige Гinstallation d'un pneumo-péritoine, c'est-à-dire d'une pression intra-abdominale exagérée, capable de perturber l'équilibre tensionnel des voies biliaires. Ce même reproche s'adresse d'ailleurs à la radiomanométrie per-opératoire, la laparotomie exerçant un déséquilibre de sens oppose.

L'inocuité des ponctions-biopsies hépatiques engagea M. Kapandjí à tenter å-’atteindre la vésicule en traversant le foie. La seule condition locale de cette audacieuse exploration est la visibilité radiologique de la vésicule; Гexamen commence en effet par un cholécystogramme de face et de profil. Une fois $\Gamma$ organe bien repéré, on pratique la ponction sur son corps, qui est fixe sous le foie (et non sur le bas-fond, qui est mobile), en évitant les incidences obliques, au moyen d'une aiguille de 1,8 à $2 \mathrm{~mm}$. de diamètre.

Une fois l'aiguille en place, on pratique la radio-manométrie; puis on vide aussi complètement que possible la vésicule, et y introduit quelques centimetres cubes d'air avant de retirer l'aiguille. La ponction transpariéto-hépatique permet de recueillir de la bile dans des conditions «normales», aux diverses phases du cycle vésicu-laire; de mesurer la capacité vésiculaire et sa pression physiologique; de mettre en evidence les dyskinésies du cholécyste et de son col; de contrôler les pressions nécessaires pour forcer les sphincters collique et cholédocien; enfin, avantage essentiel, cette exploration peut être répétée à plusieurs reprises, ne serait-ce que pour contrôler le résultat d'une thérapeutique médicamenteuse ou chirurgicale. 